\title{
Changes in Macular Perfusion Detected by Optical Coherence Tomography Angiography Following 3 Intravitreal Monthly Bevacizumab Injections for Diabetic Macular Edema in the IMPACT Study
}

\section{Ayman Elnahry ( $\square$ ayman_elnahri@hotmail.com )}

Cairo University Kasr Alainy Faculty of Medicine https://orcid.org/0000-0001-5148-316X

Ahmed A. Abdel-Kader

Cairo University

Karim A. Raafat

Cairo University Kasr Alainy Faculty of Medicine

Khaled Elrakhawy

Cairo University Faculty of Medicine

\section{Research article}

Keywords: Anti-VEGF; bevacizumab; diabetic macular ischemia; macular perfusion; OCTA; vascular density

Posted Date: August 21st, 2019

DOI: https://doi.org/10.21203/rs.2.13161/v1

License: (9) This work is licensed under a Creative Commons Attribution 4.0 International License. Read Full License

Version of Record: A version of this preprint was published at Journal of Ophthalmology on April 27th, 2020. See the published version at https://doi.org/10.1155/2020/5814165. 


\section{Abstract}

Background This study was done to evaluate macular perfusion changes following intravitreal bevacizumab injections for diabetic macular edema (DME) using spectral domain optical coherence tomography angiography (SD-OCTA). Methods This study was a prospective noncomparative interventional case series. Treatment naïve patients with DME were recruited at Cairo University retina clinic and underwent full ophthalmological examination and SD-OCTA scanning at baseline and after 3 intravitreal bevacizumab injections. Both the $6 \mathrm{X} 6$ and $3 \times 3-\mathrm{mm}$ macular scan protocols were used. Pre and post-treatment OCTA images were automatically aligned using a commercially available retina alignment software (i2k Align Retina software) then the fractal dimension (FD), vascular density (VD), and skeleton VD changes were obtained at the full retinal thickness (Full) and superficial (SCP) and deep (DCP) capillary plexuses after processing images using a semi-automated program. The foveal avascular zone (FAZ) was manually measured and FD was calculated using the FracLac plugin of ImageJ. Results Forty eyes of 26 patients were included. Following injections, there was an $8.1 \%$ increase in FAZ, 1.3\% decrease in FD-Full and FD-SCP, 1.9\% decrease in FD-DCP, 8\% decrease in VD-Full, 9.1\% decrease in VDSCP, $10.6 \%$ decrease in VD-DCP, $13.3 \%$ decrease in skeleton VD-Full, $12.5 \%$ decrease in skeleton VD-SCP, and $16.3 \%$ decrease in skeleton VD-DCP in the 6 X6 mm macular area, and a $2.6 \%$ decrease in FD-Full, $3.4 \%$ decrease in FD-SCP, $11.5 \%$ decrease in VD-Full, $14.3 \%$ decrease in VD-SCP, and $25.1 \%$ decrease in skeleton VD-SCP in the $3 X 3 \mathrm{~mm}$ macular area which were all statistically significant $(p<0.05)$. There was also an $8.3 \%$ increase in the FAZ, $18.5 \%$ decrease in skeleton VD-Full, 2.6\% decrease in FD-DCP, 8.8\% decrease in VD-DCP, and 19.7\% decrease in skeleton VD-DCP in the $3 \times 3 \mathrm{~mm}$ macular area but these were not statistically significant ( $p>0.05$ ). Using univariate and multivariate analysis, the pre-treatment $F D, V D$, and skeleton VD at each capillary layer significantly negatively correlated with the change in FD, VD, and skeleton VD at the corresponding capillary layer respectively $(p>0.05)$. Conclusion OCTA is a useful noninvasive tool for quantitative evaluation of macular perfusion changes following DME treatment. Trial Registration Trial registered at ClinicalTrials.gov on August 11, 2017 (Study ID: NCT03246152).

\section{Background}

The prevalence of diabetes mellitus (DM) has progressively increased worldwide in recent years and is expected to grow to 430 million by $2030 .{ }^{1}$ In the United States, an estimated $29 \%$ of adults with DM have diabetic retinopathy (DR) and $3 \%$ have diabetic macular edema (DME), ${ }^{2}$ with DM being the leading cause of blindness in people between the ages of 20 and $74 .{ }^{3}$ In Egypt, many patients are found to have DM and in a study of Egyptian patients with DM, diabetic retinopathy was present in $42 \%$ and legal blindness in $5 \%{ }^{4}$ This was higher than the worldwide prevalence rate of DR in diabetic patients which is estimated at $34.6 \% .^{3}$

DME is the commonest cause of loss of vison in diabetics that occurs due to accumulation of fluid in the central macular region following dysfunction of the blood retinal barrier. ${ }^{5}$ This occurs due to release of various inflammatory cytokines and growth factors, most importantly vascular endothelial growth factor 
(VEGF), following chronic hyperglycemia. ${ }^{6}$ Several treatment modalities are available for DME including intravitreal injection of anti-VEGF drugs such as off-label intravitreal bevacizumab injections. ${ }^{7}$ Because the therapeutic effect of these drugs is only temporary, however, long-term treatment is often required which raises concerns for worsening of macular ischemia and visual function due to chronic suppression of VEGF that could result in increased capillary drop-out and inhibition of reperfusion of occluded capillaries. $^{8,9}$

Most of the studies evaluating the effect of repeated intravitreal anti-VEGF injections on the macular perfusion status in diabetics have relied on qualitative analysis of perfusion using fluorescein angiography (FA).$^{10-12}$ Optical coherence tomography angiography (OCTA) is a relatively new noninvasive modality capable of analyzing the different retinal capillary layers quantitatively at a higher resolution compared to conventional FA. ${ }^{13}$ The Evaluation of the Effect of Intravitreal Injections of AntiVEGF on Macular Perfusion in Diabetic Patients using OCTA (IMPACT) study was a prospective noncomparative interventional case series done to evaluate the effect of repeated intravitreal bevacizumab injections on the macular perfusion of diabetic patients using OCTA.

\section{Methods}

\section{Study Purpose and Design:}

The IMPACT study was a prospective non-comparative interventional case series done at Cairo University Hospital to evaluate the effect of three intravitreal monthly bevacizumab injections on the macular perfusion of diabetic patients with diabetic macular edema using OCTA.

\section{Ethical Approval and Study Registration:}

Study approval was obtained from Cairo University Research Ethics Committee and the study followed the tenets of the Declaration of Helsinki. A clinical trial registration was done at ClinicalTrials.gov on August 11, 2017 before beginning patient recruitment (Study identifier: NCT03246152). Written informed consent was obtained from all study participants before inclusion in the study.

\section{Study Outcome Measures:}

The primary outcome measures of the study were the change in the FAZ area, fractal dimension, vascular density, and skeletonized vascular density following the injections. The secondary outcome measure was the change in corrected distance visual acuity following injections. Although the change in microaneurysms appearance and number was a predefined secondary outcome measure in the study, this analysis was later abandoned due to the low sensitivity of OCTA in detecting microaneurysms.

\section{Recruitment of Patients:}


Diabetic patients attending the retina clinic of Cairo University Hospital between October 2017 and September 2018 underwent comprehensive history taking and ophthalmological examination including corrected distance visual acuity (CDVA), intraocular pressure (IOP) measurement, anterior segment, and posterior segment examination. Diabetic patients who were 18 years or older and complaining of blurring of vision with a CDVA of less than 20/30 and evidence of macular edema on clinical examination underwent spectral domain optical coherence tomography (SD-OCT) (Optovue, Inc., Fremont, California, USA) and FA (TRC 50DX, Topcon, Tokyo, Japan). Patients with center involving diabetic macular edema on SD-OCT with a central macular thickness (CMT) of more than or equal to $275 \mu \mathrm{m}$ were recruited for the study. Exclusion criteria included concomitant ocular conditions that may affect retinal perfusion such as branch or central retinal vein occlusion and uveitis, history of vitreoretinal surgeries, history of previous intravitreal injections or macular laser treatment for diabetic macular edema, high myopia (refraction $\leq-6 \mathrm{D}$ ), presence of an epiretinal membrane or vitreomacular traction, significant media opacities, uncontrolled glaucoma, and systemic thromboembolic events within the last 6 months.

\section{Recruited Patients:}

Patients who were enrolled in the study then underwent a baseline macular SD-OCTA scan (Optovue, Inc., Fremont, California, USA) before starting bevacizumab injections. Each patient then underwent 3 consecutive monthly intravitreal bevacizumab injections.

Patients were instructed to follow up closely with an internist to tightly control their blood sugar, blood lipids, and blood pressure during the study period, and an $\mathrm{HbA} 1 \mathrm{C}$ measurement was obtained at the time of the last injection to determine the level of blood sugar control during the treatment period.

Patients were followed up 1 day, 1 week, and 1 month at the retina clinic following the injections and one month following the third intravitreal injection a final full ophthalmological examination was performed and documented and a follow-up OCTA scan was done using the same pretreatment protocol.

\section{Acquisition of OCTA Images:}

The AngioVue software of the RTVue XR Avanti SD-OCTA (Optovue, Inc, Fremont, CA), which uses a splitspectrum amplitude decorrelation angiography (SSADA) algorithm to improve signal-to-noise ratio, was used. The device operates at a wavelength of approximately $840 \mathrm{~nm}$ and obtains 70,000 A-scans per second. The OCTA volume scan consisted of 2 repeated B-scans from 304 sequentially uniformly spaced locations. Each B-scan consisted of 304 A-scans with an axial resolution of about $5 \mu \mathrm{m}$. The resultant three-dimensional OCTA flow data in the full retinal thickness (Full) and the superficial (SCP) and deep (DCP) capillary plexuses were projected into two-dimensional en-face OCTA images that were used in the study. Automated segmentation was performed by the machine as follows: The full retinal thickness (Full) slab was segmented from the internal limiting membrane (ILM) to $9 \mu \mathrm{m}$ below the outer plexiform layer (OPL)-outer nuclear layer (ONL) junction, while the SCP was segmented from the ILM to $9 \mu \mathrm{m}$ above 
the inner plexiform layer (IPL)-inner nuclear layer (INL) junction, and the DCP was segmented from $9 \mu \mathrm{m}$ above the IPL-INL junction to $9 \mu \mathrm{m}$ below the OPL-ONL junction. Two scan protocols, the $6 \mathrm{X} 6 \mathrm{~mm}$ and the $3 \times 3 \mathrm{~mm}$ macular scans, which were centered on the fovea, were used. The 6 X6 mm macular scan protocol was done for all included patients while the $3 \times 3 \mathrm{~mm}$ macular scan protocol was done for a group of randomly selected patients. Both scanning protocols were used since the $6 \times 6 \mathrm{~mm}$ scan allowed imaging of the perifoveal capillary network while the $3 X 3 \mathrm{~mm}$ scan provided a higher resolution of the foveal avascular zone (FAZ) and the parafoveal capillary network.

To ensure the acquisition of only high-quality images, scans were repeated if there was an insufficient signal strength index ( $\left.\mathrm{SSl}^{\prime}<5\right)$, presence of 1 or more blink artifacts, poor fixation resulting in motion or doubling artifacts, areas of localized signal loss from media opacity obscuring the vasculature, or major segmentation errors. If following repeated scans one of the aforementioned criteria persisted, the patient was excluded from the study. Minor segmentation errors were corrected manually with the built-in machine software using the corresponding structural OCT B-scans as a guide for the placement of the 2 parallel segmentation lines at the appropriate depths.

\section{Image alignment and registration:}

Post-treatment OCTA images (following 3 injections) of all patients were automatically registered and aligned with the corresponding pre-treatment images at each capillary layer using a commercially available retina alignment software (i2k Align Retina software (DualAlign, LLC, Clifton Park, NY)). ${ }^{14}$ The intersection option of the software was used to align the images which allowed comparison of only areas common to both pre and post-treatment images (Figure 1). This was necessary since the macular area imaged in the pre and post-treatment scans was not automatically registered during image acquisition by the built-in machine software.

\section{Measurement of the Foveal Avascular Zone (FAZ) Area (Primary Outcome Measure):}

The FAZ area was measured for each patient before and after the 3 injections and compared. The pre and post-treatment full retinal thickness images were used, and measurements were performed manually in all patients by a single investigator (A. G. E.) using the freehand tool of ImageJ (National Institutes of Health, Bethesda, Maryland, USA) (Figure 2). Images were scaled to millimeter units before measurements then three measurements were obtained for each image and an average value was calculated. Pre and post-treatment FAZ area measurements were compared for each patient and the FAZ change was documented.

\section{Measurement of the Vascular Density (VD) and the Fractal Dimension (FD) (Primary Outcome Measure):}


In order to quantify the changes in VD and FD following treatment, all aligned pre and post-treatment images were semi-automatically processed and quantified using a previously described and validated method. ${ }^{15,16}$ Briefly, processing and quantification of images was done as follows: En-face OCTA images were converted into a binary image by using a combined 3-way method consisting of a global threshold, hessian filter, and adaptive threshold in MATLAB (MathWorks, Inc., Natick, MA, USA). First, an area within the foveal avascular zone was manually selected 3 times with a fixed radius circle (50 pixels) to establish a baseline signal-to-noise ratio for the global thresholding step (Figure 3A, 3D, and 3G). This was the only manually performed step in the process and was done by the same investigator for all images for consistency (A. G.E). The image was then processed using a top-hat filter. This image was then automatically processed to create two separate binarized images: one created with the application of hessian filter, and another created through median adaptive thresholding. Finally, the resulting two binarized vessel maps obtained were combined to form the final binarized image (Figure 3B, 3E, and 3H). Only pixels that were detected using both the hessian filter and adaptive thresholding were included in the final binarized image. After acquiring the binary image, VD was calculated as the total image area occupied by the detected OCTA signal (white pixels in the binarized image) compared to total area of retina (total number of pixels in image). Skeletonized images were also created by deleting the pixels in the outer boundary of the binarized, white-pixelated vessels until only 1 pixel remained along the width of the vessels (Figure 3C, 3F, and 3I). VD was calculated for the skeletonized images as described for the binarized images.

Fractal Dimension (FD) which provides a global index for the branching complexity of the retinal capillary network was calculated for the skeletonized images using the FracLac plugin of ImageJ with the boxcounting method. Values were calculated for all pre and post-treatment images and compared for each patient.

\section{Statistical analysis}

Data were coded and entered using the Statistical Package for the Social Sciences (SPSS) version 25 (IBM, Chicago, IL, USA). Data was summarized using mean, standard deviation, median, minimum and maximum in quantitative data and using frequency (count) and relative frequency (percentage) for categorical data. Comparisons between quantitative variables were done using the non-parametric Kruskal-Wallis and Mann-Whitney tests. For comparison of serial measurements within same patients paired t test was used in normally distributed quantitative variables while non-parametric Wilcoxon signed rank test was used for non-normally distributed quantitative variables. Correlations between quantitative variables were done using Pearson or Spearman correlation coefficient. Linear regression analysis was done to predict changes in different parameters using baseline data. P-values less than 0.05 were considered as statistically significant.

\section{Results}




\section{Patient Demographics:}

A total of 52 eyes of 31 diabetic patients were found to be eligible and were enrolled in the study in the period between October 2017 and September 2018. Six eyes of 3 patients were lost to follow up or did not adhere to the study protocol and so were excluded from the study, while 6 eyes of 4 patients were excluded due to insufficient OCTA image quality in either the pre or post-treatment scan. Forty eyes of 26 patients successfully completed the study with the $6 \times 6 \mathrm{~mm}$ scan protocol, while 9 eyes of 6 patients successfully completed the study with the $3 X 3 \mathrm{~mm}$ scan protocol, with sufficiently high pre and posttreatment OCTA image quality. All patients who completed the study with the $3 \times 3 \mathrm{~mm}$ scan protocol also completed the study with the $6 \times 6 \mathrm{~mm}$ scan protocol. Demographic data for patients who successfully completed the study using the 6 X6 or $3 \times 3 \mathrm{~mm}$ scan protocol are shown in Table 1.

All patients who successfully completed the study received 3 intravitreal monthly bevacizumab injections. Intraoperative and postoperative complications associated with the injections included mild subconjunctival hemorrhage or transient floaters in some cases, with no major complications related to injections occurring in the study.

\section{Quantitative Analysis and Comparison of Pre and Post-Treatment Data:}

Table 2 summarizes the quantitative analysis of the pre and post-treatment results of the $6 \mathrm{X} 6 \mathrm{~mm}$ scan group while Table 3 summarizes the quantitative analysis of the pre and post-treatment results of the $3 \times 3 \mathrm{~mm}$ scan group.

In the 6 X6 mm scan group, there was a statistically significant increase in the size of the FAZ area and a statistically significant decrease in the FD, VD, and skeleton VD of Full, SCP, and DCP (Table 2, Figure 4).

In the $3 X 3 \mathrm{~mm}$ scan group, there was an increase in the size of the FAZ area, but this was not statistically significant. There was a statistically significant decrease in the FD and VD Full and SCP, and in the skeleton VD-SCP (Figure 4). There was a decrease in skeleton VD-Full, and FD, VD, and skeleton VD-DCP but these were not statistically significant (Table 3, Figure 4).

\section{Correlations between Baseline Data (6X6 protocol only):}

In the 6 X6 group, pre-treatment logMAR CDVA significantly positively correlated with pre-treatment CMT ( $r$ $=0.617, p<0.001)$, pre-treatment parafoveal thickness $(r=0.660, p<0.001)$, and severity of retinopathy $(r$ $=0.357, p=0.024)$. The severity of retinopathy positively correlated with pre-treatment CMT $(r=0.354, p$ $=0.025)$ but negatively correlated with pre-treatment FD-SCP $(r=-0.353, p=0.026)$, pre-treatment FD$\operatorname{DCP}(r=-0.378, p=0.016)$, pre-treatment VD-DCP $(r=-0.315, p=0.048)$, pre-treatment skeleton VD-SCP $(r=-0.35, p=0.027)$, and pre-treatment skeleton VD-DCP $(r=-0.40, p=0.011)$. 


\section{Factors associated with post-treatment changes in Fractal Dimension (6X6 protocol only):}

Using univariate analysis with Spearman's rank test, there was a statistically significant negative correlation between pre-treatment FD-Full and the change in FD-Full $(r=-0.541, p<0.001)$ (Figure 5A), pre-treatment FD-SCP and the change in FD-SCP $(r=-0.546, p<0001)$, the change in CMT and the change in FD-SCP $(r=-0.324, p=0.042)$ (Figure 5B), and pre-treatment FD-DCP and the change in FD$\mathrm{DCP}(r=-0.345, p=0.029)$. The severity of retinopathy at baseline positively correlated with the change in FD-DCP only $(r=0.345, p=0.029)$.

Using multivariate analysis, there was a significant negative correlation between pre-treatment FD-Full and the change in FD-Full ( $p<0.001)$, pre-treatment FAZ area and the change in FD-Full $(p=0.039)$, pretreatment FD-SCP and the change in FD-SCP $(p<0001)$, pre-treatment FD-DCP and the change in FD-DCP $(p<0.001)$, and pre-treatment FAZ area and the change in FD-DCP $(p=0.044)$.

\section{Factors associated with post-treatment changes in Vascular Density (6X6 protocol only):}

Using univariate analysis with Spearman's rank test, there was a statistically significant negative correlation between pre-treatment VD-Full and the change in VD-Full $(r=-0.560, p<0.001)$ (Figure 5C), pre-treatment VD-SCP and the change in VD-SCP $(r=-0.497, p=0001)$, the change in CMT and the change in VD-SCP $(r=-0.389, p=0.013)$ (Figure 5D), and pre-treatment VD-DCP and the change in VDDCP $(r=-0.441, p=0.004)$. The CMT at baseline positively correlated with the change in VD-SCP only $(r$ $=0.319, p=0.045)$.

Using multivariate analysis, there was a significant negative correlation between pre-treatment VD-Full and the change in VD-Full $(p<0.001)$, pre-treatment FAZ area and the change in VD-Full $(p=0.017)$, pretreatment skeleton VD-SCP and the change in VD-SCP $(p<0001)$, hypertension and the change in VD-SCP $(p=0.001)$, type of DM and the change in VD-SCP $(p=0.01)$, pre-treatment FAZ area and the change in $\operatorname{VD-SCP}(p=0.005)$, and pre-treatment FD-DCP and the change in VD-DCP $(p=0.001)$.

\section{Factors associated with post-treatment changes in Skeleton Vascular Density (6X6 protocol only):}

Using univariate analysis with Spearman's rank test, there was a statistically significant negative correlation between pre-treatment skeleton VD-Full and the change in skeleton VD-Full $(r=-0.694, p<$ 0.001) (Figure 5E), pre-treatment skeleton VD-SCP and the change in skeleton VD-SCP $(r=-0.667, p<$ 0001), the change in CMT and the change in skeleton VD-SCP $(r=-0.377, p=0.016)$, and pre-treatment skeleton VD-DCP and the change in skeleton VD-DCP $(r=-0.508, p=0.001)$. The severity of retinopathy at baseline positively correlated with the change in skeleton VD-DCP only $(r=0.365, p=0.021)$. 
Using multivariate analysis, there was a significant negative correlation between pre-treatment skeleton VD-Full and the change in skeleton VD-Full $(p<0.001)$, pre-treatment skeleton VD-SCP and the change in skeleton VD-SCP $(p<0.001)$, and pre-treatment FD-DCP and the change in skeleton VD-DCP $(p<0.001)$.

\section{Discussion}

Our study was a prospective interventional case series done to evaluate the effect of three intravitreal bevacizumab injections on the macular perfusion of diabetic patients with DME using OCTA. A total of 40 eyes of 26 patients were successfully imaged before and after 3 monthly intravitreal injections using the $6 \times 6 \mathrm{~mm}$ macular scan protocol of OCTA, while 9 eyes of 6 patients were imaged using the $3 \times 3 \mathrm{~mm}$ macular scan protocol. All patients were treatment naïve at the beginning of the study and were treated with 3 intravitreal bevacizumab injections for DME during the study.

Using the $6 \times 6 \mathrm{~mm}$ scan protocol, we found an $8.1 \%$ increase in the mean size of the FAZ area, a $1.3 \%$ decrease in the mean FD-Full and mean FD-SCP, a 1.9\% decrease in the mean FD-DCP, an $8 \%$ decrease in the mean VD-Full, a 9.1\% decrease in the mean VD-SCP, a 10.6\% decrease in the mean VD-DCP, a 13.3\% decrease in the mean skeleton VD-Full, a 12.5\% decrease in the mean skeleton VD-SCP, and a 16.3\% decrease in the mean skeleton VD-DCP following 3 months of intravitreal bevacizumab injections. These changes were all statistically significant.

Using the $3 \times 3 \mathrm{~mm}$ scan protocol, we found a $2.6 \%$ decrease in the mean FD-Full, a $3.4 \%$ decrease in the mean FD-SCP, an $11.5 \%$ decrease in the mean VD-Full, a $14.3 \%$ decrease in the mean VD-SCP, and a $25.1 \%$ decrease in the mean skeleton VD-SCP following 3 monthly intravitreal bevacizumab injections that were all statistically significant. There was also an $8.3 \%$ increase in the mean size of the FAZ area, a $2.6 \%$ decrease in the mean FD-DCP, an $8.8 \%$ decrease in the mean VD-DCP, an $18.5 \%$ decrease in the mean Skeleton VD-Full, and a 19.7\% decrease in the mean Skeleton VD-DCP. These changes, however, were not statistically significant, possibly due to the smaller number of eyes imaged using the $3 \times 3 \mathrm{~mm}$ protocol.

In the BOLT study, a prospective study that compared the efficacy of 6 weekly intravitreal bevacizumab injections to 4-monthly modified ETDRS macular laser treatment, there was no evidence of worsening of macular ischemia in either group at 4 months after treatment initiation. A total of 40 eyes were evaluated in the bevacizumab group. This study, however, used fluorescein angiography to analyze changes in the macular perfusion, evaluated changes only related to the fovea, and assessed the status of perifoveal capillaries qualitatively using human graders. ${ }^{10}$

In an analysis of the RIDE and RISE studies, 2 parallel prospective multicenter trials comparing 2 doses of ranibizumab $(0.3$ and $0.5 \mathrm{mg})$ to sham injections, there was worsening of posterior retinal nonperfusion in all groups but more in the sham injected group. The authors concluded that monthly injections of ranibizumab can thus slow, but not completely prevent, retinal capillary closure in patients with DME. Again, this study depended on FA images for the pre and post-treatment analyses and used trained 
human graders. ${ }^{11}$ In this study, the authors hypothesized that worsening of retinal nonperfusion in patients with DME could be the result of VEGF-induced leukostasis that was partially reversed by the antiVEGF therapy. ${ }^{11,17}$ In an electron microscopical investigation of retinal capillaries in VEGF-A-induced retinopathy in monkeys, however, there was no leukocytes adherent to the vascular wall. ${ }^{18}$ This study, however, identified endothelial cell hypertrophy that is induced by local VEGF-A production as another possible cause of VEGF-induced worsening of capillary nonperfusion by causing progressive luminal narrowing. ${ }^{18}$

In another study analyzing the effect of aflibercept for DME on the macular perfusion (analysis of VISTA patients), there was both more improvement in retinal non perfusion and slowing of worsening retinal non-perfusion with aflibercept compared to laser treatment. ${ }^{12}$ This study, however, also used fluorescein angiography and human graders.

OCTA is a new non-invasive modality that is capable of resolving the different retinal vascular layers separately to the capillary level at a higher resolution compared to conventional FA. ${ }^{13,19,20}$ It has the ability to precisely and reliably delineate areas of capillary drop-out and to image the FAZ without obscuration by dye leakage compared to FA. ${ }^{19,20}$ It allows quantitative measurements of the vascular density and fractal dimension reliably and reproducibly in the macular area in an objective manner that does not require human graders. ${ }^{16,21,22}$ Furthermore, unlike FA, OCTA does not require intravenous dye injection which has been associated with side effects including nausea, vomiting, and anaphylaxis. ${ }^{23}$ This allows OCTA to be repeated frequently with no risks or discomfort to the patient. These advantages may allow OCTA to be more suited for the analysis of changes in macular perfusion following DME treatment. $^{24}$

In a study investigating the effect of aflibercept injections for treatment naïve wet age-related macular degeneration on the macular vascular density using OCTA, there was a statistically significant decrease in the superficial foveal and parafoveal vascular density after 1,2, and 3 injections compared to baseline (approximately $31 \%$ reduction in foveal vascular density and $6.6 \%$ reduction in parafoveal vascular density after 3 injections). ${ }^{25}$ This is interesting since patients with a history of retinal vascular disease were excluded from the study which suggests that anti-VEGF injections may affect the vascular density of normal retinal vessels. The automated measurements of the AngioVue software were used in this study and the authors hypothesized that aflibercept may result in decreased vascular density as measured by OCTA due to either decrease in nitric oxide, leading to vasoconstriction, or capillary rarefaction, which was described in experimental mouse models treated with VEGF inhibitor. ${ }^{25,26}$ Limitation of this study included the small number of included eyes (15 eyes) and the lack of a control group.

OCTA was also used to evaluate the effect of a single anti-VEGF injection on the macular perfusion in patients with macular edema secondary to DR or central retinal vein occlusion (CRVO) in a prospective non-comparative case series. ${ }^{27}$ This study found that the FAZ area and the foveal and parafoveal vessel 
density of the SCP and DCP did not significantly change after the single injection. Further analysis of data from the study, however, revealed an increase in the size of the FAZ and a decreased in the vascular density of the foveal area following the single injection which, however, was not statistically significant. This may be due to the relatively small number of eyes included in the study (18 eyes), the variability in the anti-VEGF agent used (bevacizumab in 14 eyes, aflibercept in 3 eyes, and ranibizumab in 1 eye), the inclusion of 2 different causes of macular edema (DME in 13 eyes and CRVO in 5 eyes), the short duration of treatment (1 month), and the use of vascular density measurements from the machine software. The study also did not exclude patients with a history of previous anti-VEGF injections which may have influenced the results.

In another retrospective study evaluating the effect of repeated intravitreal anti-VEGF injections for treatment of DME or PDR on the macular perfusion using OCTA $3 \times 3 \mathrm{~mm}$ and $6 \times 6 \mathrm{~mm}$ scan protocols, there was no statistically significant difference in the macular perfusion after 1, 2, and 3 injections using both scanning protocols. ${ }^{28}$ Although the study included 46 eyes which performed OCTA after the first injection, only 28 and 26 eyes performed OCTA after the $2^{\text {nd }}$ and $3^{\text {rd }}$ injections respectively. Unlike our study, the type of anti-VEGF agent used was variable ( $45.7 \%$ bevacizumab, $42.4 \%$ aflibercept, and $11.9 \%$ ranibizumab) and the mean interval between injections was 47 days. Half of the eyes (23 eyes) that performed OCTA after the $1^{\text {st }}$ injection were not treatment naïve. Quantification of the vascular density was performed automatically using the proprietary built-in machine software (AngioVue software) using the ETDRS grid.

Commercially available OCTA is currently limited to evaluating the perfusion of the macular area but the technology is rapidly evolving. Using ultrawide field imaging, anti-VEGF injections were found to be associated with improved DR severity score (DRSS) on ultrawide field color fundus photographs but with no evidence of reperfusion of either arterioles or venules in or around non-perfusion areas on ultrawide field FA following 3 monthly injections. ${ }^{29}$ On the contrary, following the last injection, $83 \%$ of studied eyes showed occlusion of few vessel segments (mean: $6 \pm 11$ per eye) passing through the non-perfusion areas. Interestingly, similar to our study, this study also used i2k Align retina software to align pre and post-treatment images allowing only areas common to both images to be compared. Future technology enabling ultrawide field OCTA imaging can help confirm these results using the advantages of OCTA imaging. ${ }^{30}$

An important factor that could influence the measurements of vascular density using OCTA is the scan signal strength index (SSI). In a study of the effect of signal strength on OCTA metrics, differences in signal strength were associated with statistically significant differences in measurements of vascular density, perfusion density, and FAZ metrics with increased signal strength being associated with a significantly increased value of all measurements. ${ }^{31}$ Consideration of this data is essential for correct analysis of OCTA data. In our study, there was no significant difference between pre and post-treatment imaging signal strength, which further supports the validity of our results. 
Another factor that could also influence OCTA measurements is IOP. In a study evaluating macular and peripapillary vascular density changes immediately following an intravitreal anti-VEGF injection for various pathologies, there was a statistically significant decrease in angiographic perfusion density in most areas of the superficial and deep layer macular vascular density, with more affection of the superficial layer, and the overall optic nerve head and the radial peripapillary capillary layer, preferentially temporal. ${ }^{32}$ In that study, the mean pre-treatment IOP was $17.15 \mathrm{mmHg}$ while the mean immediate posttreatment IOP, taken approximately 15 seconds after the injection, was $46.35 \mathrm{mmHg}$ as measured by Tonopen (Reichert, Depew, NY). The superficial macular density decreased by $7.8 \%$ while the deep macular density decreased by $3.5 \%$ immediately (within 3 minutes) following the injection. The authors hypothesized that the acute rise in IOP was the cause of reduced vascular density observed in the study. In our study, there was no statistically significant difference between pre and post-treatment IOP suggesting that IOP did not have a significant effect on our measurements.

An increase in the FAZ size following intravitreal bevacizumab injections for DME has also been previously reported using FA in several noncomparative studies. ${ }^{33,34}$ In one of these studies, there was a $19.7 \%$ increase in the FAZ area 6-8 weeks following a single bevacizumab injection for $D M E,{ }^{33}$ while another study showed a $13 \%$ increase in the FAZ area following 3 monthly bevacizumab injections which was greater in patients with milder DR. ${ }^{34}$ This was in agreement with our results and could be due to progression of ischemia due to the underlying DR or due to VEGF inhibition or both. ${ }^{34}$ Controlled studies are needed to further explain these findings.

Several explanations could account for our findings. First, although long term treatment with anti-VEGF agents have been shown to improve the DRSS, studies have shown that they are not able to completely halt the progressive retinal capillary closure associated with DR but to only slow it down compared to sham or laser. ${ }^{11,12,29}$ This was consistent with our findings where we found a significant decrease in retinal vascular density associated with anti-VEGF injections, however, whether this decrease could have been more in the absence of injections or using other treatment modalities could not be assessed in our study due to the absence of a control group. Second, in a study using doppler ultrasonography, there was a statistically significant decrease of $10 \%, 20 \%$, and $20 \%$ in the mean blood flow velocity of the central retinal artery, temporal posterior ciliary arteries, and ophthalmic arteries, respectively, 4 weeks following a single intravitreal bevacizumab injection in patients with wet age-related macular degeneration. ${ }^{35}$ The authors concluded that bevacizumab may result in hypoperfusion of the whole globe through vasoconstriction and decrease in capillary density. This could explain the decreased vascular density and flow detected by OCTA following bevacizumab injections in our study. Third, in a recent study of eyes with DME, both ranibizumab and bevacizumab injections were found to result in a significant constriction in the retinal blood vessels diameter measured using a semiautomated system. ${ }^{36}$ In that study, following a single injection of treatment naïve eyes, the central retinal artery diameter decreased by $4.5 \%$ and the central retinal vein diameter decreased by $7.8 \%$ in the ranibizumab group, while the central retinal artery diameter decreased by $2.9 \%$ and the central retinal vein diameter decreased by $3.2 \%$ in the bevacizumab group one month after the injection. There was no change in the vessel diameter in the untreated control 
group. This effect maybe due to inhibition of nitric oxide following VEGF inhibition which results in vasoconstriction and possible decrease in retinal blood flow which may explain the decreased vascular density and flow observed following bevacizumab injections in our study. Fourth, VEGF-A plays an important role in both physiological vascular development and pathological neovascularization in the retina and choroid, and the retinal vessels are initially dependent on VEGF as a survival factor, ${ }^{37}$ but such dependence is lost as soon as capillaries are covered by pericytes. ${ }^{38,39}$ In DR, there is early loss of pericytes ${ }^{40}$ and this may perhaps render capillary endothelial cells in patients with DR susceptible to antiVEGF inhibition, ${ }^{41}$ leading to endothelial apoptosis and decreased vascular density.

Using univariate and multivariate analysis, we have found that pre-treatment FD, VD, and skeleton VD at each capillary layer significantly negatively correlate with the change in FD, VD, and skeleton VD at the corresponding capillary layer respectively. This may be due to a floor effect, where eyes with more ischemia show less change over time due to a lower baseline vascularity as previously suggested. ${ }^{34}$

Strengths of our study include the prospective nature of the study, the strict exclusion criteria including previous treatment for macular edema, inclusion of patients with a single diagnosis (DME), use of a single type of anti-VEGF agent, use of 2 scanning protocols, use of automated image alignment for pre and post-treatment images, and the previously unreported analysis of post-treatment changes in fractal dimension, a measure of complexity and branching of retinal vessels, and skeleton vascular density, which is a more sensitive approach for estimation of retinal non-perfusion. ${ }^{16}$ Limitations of our study include the relatively small number of patients, inclusion of both eyes of some patients, the relatively short period of treatment, and the absence of a control group. Having a proper control group, however, could be difficult, as most patients with DME benefit from, and are currently being treated at least initially with, anti-VEGF agents. In the light of our findings, however, having a sham control group in a future study may seem proper for at least a short-term period. Several imaging artifacts are currently associated with OCTA imaging and may have influenced our results, ${ }^{42,43}$ however, we have taken several measures to limit the effects of these artifacts on the results of our study by: excluding patients with media opacities, repeating scans until no major obvious artifacts were present in the image, excluding patients with persisting major artifacts, including only images with an SSI higher than 5, manually correcting minor segmentation errors, using full thickness retinal slabs which are less affected by edema and segmentation errors, and using complex thresholding and filtering processes to create the final binarized images.

\section{Conclusion}

In conclusion, our study showed that using OCTA there was a significant increase in the FAZ area and a significant decrease in the fractal dimension and vascular density associated with three monthly intravitreal bevacizumab injections for the treatment of DME. The significance of this, however, is unclear since our patients still showed significant improvement in CDVA and CMT with only changes in the SCP showing a significant negative correlation with changes in CMT. Several possible mechanisms could 
explain our findings as we have discussed but more studies using OCTA, preferably in the form of large randomized controlled trials, are needed to further confirm and explain the findings of this study.

\section{Abbreviations}

CMT: central macular thickness; CDVA: corrected distance visual acuity; DCP: deep capillary plexus; DM: diabetes mellitus; DME: diabetic macular edema; DR: diabetic retinopathy; FA: fluorescein angiography; FAZ: foveal avascular zone; FD: fractal dimension; Full: full retinal thickness; HbA1C: hemoglobin A1C; ILM: internal limiting membrane; INL: inner nuclear layer; IOP: intraocular pressure; IPL: inner plexiform layer; OCT: optical coherence tomography; OCTA: optical coherence tomography angiography; ONL: outer nuclear layer; OPL: outer plexiform layer; SCP: superficial capillary plexus; SD: spectral domain; SSADA: split spectrum amplitude decorrelation angiography; SSI: signal strength index; VD: vascular density; VEGF: vascular endothelial growth factor.

\section{Declarations}

\section{Ethics approval and consent to participate}

This study was approved by Cairo University Research Ethics Committee (Study ID: N-79-2017) and followed the tenets of the Declaration of Helsinki. All patients signed a written informed consent before inclusion in the study.

\section{Consent for publication}

Signed consent forms for publication of patients' OCTA images were obtained.

\section{Availability of data and material}

The datasets used and/or analysed during the current study are available from the corresponding author on reasonable request.

\section{Competing interests}

The authors declare that they have no competing interests.

\section{Funding}

There are no sources of funding.

\section{CONSORT guidelines}


This study adheres to the CONSORT guidelines for reporting clinical trials.

\section{Authors' contributions}

AGE has made substantial contribution in the conception and design of the work, and in acquisition, analysis, and interpretation of the data, and in drafting the manuscript. AAA has contributed to the acquisition, analysis, analysis, and interpretation of the data, and in revising the manuscript. KAR has made substantial contribution in the conception and design of the work, and in acquisition, analysis, and interpretation of the data. KE has made substantial contribution in the conception and design of the work and in the revision of the manuscript. All authors read and approved the final manuscript.

\section{Acknowledgements}

The authors would like to thank Dr. Ruikang K. Wang, Zhongdi Chu, and Xiao Zhou (University of Washington), and Dr David J. Ramsey and Dr Khaled Elmahgoub (Tufts University) for their assistance and support with image processing techniques.

\section{References}

1. Korobelnik JF, Do DV, Schmidt-Erfurth U, Boyer DS, Holz FG, Heier JS, Midena E, Kaiser PK, Terasaki $\mathrm{H}$, Marcus DM, et al. Intravitreal aflibercept for diabetic macular edema. Ophthalmology 2014; 121:2247-54.

2. Zhang X, Saaddine JB, Chou CF, Cotch MF, Cheng YJ, Geiss LS, Gregg EW, Albright AL, Klein BEK, Klein R. Prevalence of diabetic retinopathy in the United States, 2005-2008. JAMA. 2010; 304:64956.

3. Klein BE. Overview of epidemiologic studies of diabetic retinopathy. Ophthalmic Epidemiol 2007; $14: 179-83$.

4. Herman WH, Aubert RE, Engelgau MM, Thompson TJ, Ali MA, Sous ES, Hegazy M, Badran MA, Kenny SJ, Gunter EW et al. Diabetes mellitus in Egypt: glycaemic control and microvascular and neuropathic complications. Diabetic Medicine. 1998; 15(12):1045-51.

5. Yau JW, Rogers SL, Kawasaki R, Lamoureux EL, Kowalski JW, Bek T, Chen SJ, Dekker JM, Fletcher A, Grauslund J, et al. Global prevalence and major risk factors of diabetic retinopathy. Diabetes Care 2012; 35: 556-564.

6. Yokoi M, Yamagishi SI, Takeuchi M, Ohgami K, Okamoto T, Saito W, Muramatsu M, Imaizumi T, Ohno S. Elevations of AGE and vascular endothelial growth factor with decreased total antioxidant status in the vitreous fluid of diabetic patients with retinopathy. $\mathrm{Br} \mathrm{J}$ Ophthalmol 2005; 89(6):673-5.

7. Bahrami B, Zhu M, Hong T, Chang A. Diabetic macular oedema: pathophysiology, management challenges and treatment resistance. Diabetologia 2016; 59(8):1594-608. 
8. Manousaridis K, Talks J. Macular ischaemia: a contraindication for anti-VEGF treatment in retinal vascular disease? Br J Ophthalmol. 2012;96(2):179-84.

9. Elnahry AG, Abdel-Kader AA, Raafat KA, Elrakhawy K. Evaluation of the Effect of Repeated Intravitreal Bevacizumab Injections on the Macular Microvasculature of a Diabetic Patient Using Optical Coherence Tomography Angiography. Case Rep Ophthalmol Med. 2019; 2019: 3936168.

10. Michaelides M, Fraser-Bell S, Hamilton R, Kaines A, Egan C, Bunce C, Peto T, Hykin P. Macular perfusion determined by fundus fluorescein angiography at the 4-month time point in a prospective randomized trial of intravitreal bevacizumab or laser therapy in the management of diabetic macular edema (Bolt Study): Report 1. Retina. 2010;30(5):781-6.

11. Campochiaro PA, Wykoff CC, Shapiro H, Rubio RG, Ehrlich JS. Neutralization of vascular endothelial growth factor slows progression of retinal nonperfusion in patients with diabetic macular edema. Ophthalmology. 2014; 121:1783-1789.

12. Wykoff CC, Shah C, Dhoot D, Coleman HR, Thompson D, Du W, Baker K, Vitti R, Berliner AJ, Metzig C, et al. Longitudinal Retinal Perfusion Status in Eyes with Diabetic Macular Edema Receiving Intravitreal Aflibercept or Laser in VISTA Study. Ophthalmology. 2019; 1-10. https://doi.org/10.1016/j.ophtha.2019.03.040

13. Spaide RF, Klancnik JM, Cooney MJ. Retinal vascular layers imaged by fluorescein angiography and optical coherence tomography angiography. JAMA Ophthalmol 2015;133: 45-50.

14. Ramsey DJ, Sunness JS, Malviya P, Applegate C, Hager GD, Handa JT. Automated image alignment and segmentation to follow progression of geographic atrophy in age-related macular degeneration. Retina. 2014;

15. Reif R, Qin J, An L, Zhi Z, Dziennis S, Wang R. Quantifying optical microangiography images obtained from a spectral domain optical coherence tomography system. Int J Biomed Imaging. 2012; 2012:509783.

16. Kim AY, Chu Z, Shahidzadeh A, Wang RK, Puliafito CA, Kashani AH. Quantifying microvascular density and morphology in diabetic retinopathy using spectral-domain optical coherence tomography angiography. Invest Ophthalmol Vis Sci. 2016; 57(9): ОСT362-70.

17. Tolentino MJ, Miller JW, Gragoudas ES, Jakobiec FA, Flynn E, Chatzistefanou K, Ferrara N, Adamis AP. Intravitreous injections of vascular endothelial growth factor produce retinal ischemia and microangiopathy in an adult primate. Ophthalmology 1996; 103:1820-1828.

18. Hofman P, Van Blijswijk BC, Gaillard PJ, Vrensen GF, Schlingemann RO. Endothelial cell hypertrophy induced by vascular endothelial growth factor in the retina: new insights into the pathogenesis of capillary nonperfusion. Arch Ophthalmol. 2001;119:861-866.

19. Couturier A, Mane V, Bonnin S, Erginay A, Massin P, Gaudric A, Tadayoni R. Capillary Plexus Anomalies in Diabetic Retinopathy on Optical Coherence Tomography Angiography. Retina 2015; 35:2384-91.

20. Ishibazawa A, Nagaoka T, Takahashi A, Omae T, Tani T, Sogawa K, Yokota H, Yoshida A. Optical Coherence Tomography Angiography in Diabetic Retinopathy: A Prospective Pilot Study. Am J 
Ophthalmol. 2015; 160:35-44.e1.

21. Rabiolo A, Gelormini F, Sacconi R, Cicinelli MV, Triolo G, Bettin P, Nouri-Mahdavi K, Bandello F, Querques $\mathrm{G}$. Comparison of methods to quantify macular and peripapillary vessel density in optical coherence tomography angiography. PLoS ONE 2018;

13(10):e0205773.https://doi.org/10.1371/journal.pone.0205773

22. Lei J, Durbin MK, Shi Y, Uji A, Balasubramanian S, Baghdasaryan E, Al-Sheikh M, Sadda SR. Repeatability and Reproducibility of Superficial Macular Retinal Vessel Density Measurements Using Optical Coherence Tomography Angiography En Face Images. JAMA Ophthalmol. 2017; 135(10):1092-8.

23. Yannuzzi LA, Rohrer KT, Tindel LJ, Sobel RS, Costanza MA, Shields W, Zang E. Fluorescein angiography complication survey. Ophthalmology.1986;93(5):611-617.

24. Reddy RK, Pieramici DJ, Gune S, Ghanekar A, Lu N, Quezada-Ruiz C, Baumal CR. Efficacy of Ranibizumab in Eyes with Diabetic Macular Edema and Macular Nonperfusion in RIDE and RISE. Ophthalmology. 2018; 125:1568-1574.

25. Mastropasqua L, Toto L, Borrelli M, Carpineto P, Antonio LD, Mastropasqua R. Optical coherence tomography angiography assessment of vascular effects occuring after aflibercept intravitreal injections in treatment-naïve patients with wet age-related macular degeneration. Retina. 2017; 37:247-256.

26. Kamba T, McDonald DM. Mechanisms of adverse effects of anti-VEGF therapy for cancer. Br J Cancer 2007; 96:1788-1795.

27. Ghasemi Falavarjani K, lafe NA, Hubschman JP, Tsui I, Sadda SR, Sarraf D. Optical coherence tomography angiography analysis of the foveal avascular zone and macular vessel density after anti-VEGF therapy in eyes with diabetic macular edema and retinal vein occlusion. Invest Ophthalmol Vis Sci. 2017; 58(1):30-34.

28. Sorour OA, Sabrosa AS, Yasin Alibhai A, Arya M, Ishibazawa A, Witkin AJ, Baumal CR, Duker JS, Waheed NK. Optical coherence tomography angiography analysis of macular vessel density before and after anti-VEGF therapy in eyes with diabetic retinopathy. Int Ophthalmol. 2019. https://doi.org/10.1007/s10792-019-01076-x.

29. Bonnin S, Dupas B, Lavia C, Erginay A, Dhundass M, Couturier A, Gaudric A, Tadayoni R. Antivascular endothelial growth factor therapy can improve diabetic retinopathy score without change in retinal perfusion. Retina. 2019; 39:426-434.

30. Pellegrini M, Cozzi M, Staurenghi G, Corvi F. Comparison of wide field optical coherence tomography angiography with extended field imaging and fluorescein angiography in retinal vascular disorders. PloS one. 2019; 14(4): e0214892.

31. Lim HB, Kim YW, Kim JM, Jo YJ, Kim JY. The Importance of Signal Strength in Quantitative Assessment of Retinal Vessel Density Using Optical Coherence Tomography Angiography. Sci Rep. 2018; 8:12897. https://doi.org/10.1038/s41598-018-31321-9. 
32. Barash A, Chui TYP, Garcia P, Rosen RB. Acute macular and peripapillary angiographic changes with intravitreal injections. Retina. 2018; 1-9.

33. Feucht N, Schönbach EM, Lanzl I, Kotliar K, Lohmann CP, Maier M. Changes in the foveal microstructure after intravitreal bevacizumab application in patients with retinal vascular disease. Clin Ophthalmol. 2013; 7:173-8.

34. Erol N, Gursoy H, Kimyon S, Topbas S, Colak E. Vision, retinal thickness, and foveal avascular zone size after intravitreal bevacizumab for diabetic macular edema. Adv Ther. 2012; 29:359-69.

35. Bonnin P, Pournaras JA, Lazrak Z, Cohen SY, Legargasson JF, Gaudric A, Levy BI, Massin P. Ultrasound assessment of short-term ocular vascular effects of intravitreal injection of bevacizumab (Avastin ${ }^{\circledR}$ ) in neovascular age-related macular degeneration. Acta ophthalmol. 2010;88(6):641-5.

36. Kurt MM, Çekiç O, Akpolat C, Elçioglu M. Effects of intravitreal ranibizumab and bevacizumab on the retinal vessel size in diabetic macular edema. Retina. 2018; 38:1120-1126.

37. Alon T, Hemo I, Itin A, Pe'er J, Stone J, Keshet E. Vascular endothelial growth factor acts as a survival factor for newly formed retinal vessels and has implications for retinopathy of prematurity. Nat Med 1995; 1 : 1024-8.

38. Benjamin LE, Hemo I, Keshet E. A plasticity window for blood vessel remodelling is defined by pericyte coverage of the preformed endothelial network and is regulated by PDGF-B and VEGF. Development 1998; 125:1591-1598.

39. Lindhal P, Johansson BE, Leveen P, Betsholtz C. Pericyte loss and microaneurysm formation in PDGFB-deficient mice. Science 1997;277:242-245.

40. Stitt AW, Gardiner TA, Archer DB. Histological and ultrastructural investigation of retinal microaneurysm development in diabetic patients. Br J Ophthalmol 1995; 79(4):362-367

41. Dorrell MI, Aguilar E, Scheppke L, Barnett FH, Friedlander M. Combination angiostatic therapy completely inhibits ocular and tumor angiogenesis. Proc Natl Acad Sci USA 2007; 104(3):967-72.

42. Spaide RF, Fujimoto JG, Waheed NK, Sadda SR, Staurenghie G. Optical coherence tomography angiography. Progress in retinal and eye research. 2018; 64:1-55.

43. Spaide RF, Fujimoto JG, Waheed NK. Image artifacts in optical coherence tomography angiography. Retina. 2015; 35(11):2163-2180.

\section{Tables}

Table 1: Demographic data of included patients in both scanning protocols 


\begin{tabular}{|c|c|c|c|c|}
\hline & \multicolumn{2}{|c|}{$6 \mathrm{X} 6 \mathrm{~mm}$ protocol } & \multicolumn{2}{|c|}{$3 \mathrm{X} 3 \mathrm{~mm}$ protocol } \\
\hline Number of patients & 26 & & 6 & \\
\hline Males & 12 & $46.2 \%$ & 1 & $16.7 \%$ \\
\hline Females & 14 & $53.8 \%$ & 5 & $83.3 \%$ \\
\hline Number of eyes & 40 & & 9 & \\
\hline Males & 19 & $47.5 \%$ & 1 & $11.1 \%$ \\
\hline Females & 21 & $52.5 \%$ & 8 & $88.9 \%$ \\
\hline Age (mean \pm SD) , yrs & $54.8 \pm 6.52$ & & $51.56 \pm 6.54$ & \\
\hline DM Type (patients) & & & & \\
\hline Type 1 & 3 & $11.5 \%$ & 1 & $16.7 \%$ \\
\hline Туре 2 & 23 & $88.5 \%$ & 5 & $83.3 \%$ \\
\hline DM Duration (mean \pm SD), yrs & $16.53 \pm 6$ & & $12 \pm 6.87$ & \\
\hline HbA1C (mean \pm SD), \% & $8.07 \pm 1.77$ & & $8.8 \pm 1.48$ & \\
\hline DM Treatment (patients) & & & & \\
\hline Oral drugs & 7 & $26.9 \%$ & 3 & $50 \%$ \\
\hline Insulin & 19 & $73.1 \%$ & 3 & $50 \%$ \\
\hline Hypertension (patients) & 14 & $53.8 \%$ & 2 & $33.3 \%$ \\
\hline Retinopathy Grade (eyes) & & & & \\
\hline Mild NPDR & 8 & $20 \%$ & 3 & $33.3 \%$ \\
\hline Moderate NPDR & 14 & $35 \%$ & 3 & $33.3 \%$ \\
\hline Severe NPDR & 9 & $22.5 \%$ & 3 & $33.3 \%$ \\
\hline PDR & 9 & 22.5 & 0 & $0 \%$ \\
\hline Lens status (eyes) & & & & \\
\hline
\end{tabular}




\begin{tabular}{|l|c|c|c|c|} 
Nuclear sclerosis & 24 & $60 \%$ & 5 & $55.6 \%$ \\
Nuclear cataract +1 & 6 & $15 \%$ & 1 & $11.1 \%$ \\
Nuclear cataract +2 & 1 & $2.5 \%$ & 0 & $0 \%$ \\
Posterior subcapsular & 7 & $17.5 \%$ & 3 & $33.3 \%$ \\
Pseudophakic & 2 & $5 \%$ & 0 & $0 \%$ \\
\hline
\end{tabular}

Table 2: Quantitative analysis and comparison of the pre and post-treatment results of the 6X6 mm scan group

\begin{tabular}{|c|c|c|c|c|c|}
\hline Metric & Pre-Treatment & Post-Treatment & Change & \% Change & $p$-value ${ }^{a}$ \\
\hline LogMAR CDVA (mean \pm SD) & $0.68 \pm 0.34$ & $0.47 \pm 0.25$ & $-0.21 \pm 0.27$ & $-30.9 \%$ & $<0.001^{\mathrm{b}}$ \\
\hline CMT (mean \pm SD),$\mu \mathrm{m}$ & $422 \pm 131$ & $334 \pm 98$ & $-88 \pm 132$ & $-20.9 \%$ & $<0.001^{\mathrm{b}}$ \\
\hline PFT (mean \pm SD), $\mu \mathrm{m}$ & $413 \pm 76$ & $364 \pm 45$ & $-49 \pm 72$ & $-11.9 \%$ & $<0.001^{\mathrm{C}}$ \\
\hline IOP (mean \pm SD), mmHg & $17.6 \pm 3.1$ & $16.6 \pm 3.7$ & $-0.31 \pm 3.2$ & $-1.8 \%$ & $0.737^{\mathrm{C}}$ \\
\hline Signal Strength (mean \pm SD) & $7.13 \pm 1.57$ & $7.15 \pm 1.59$ & $0.05 \pm 1.04$ & $0.7 \%$ & $0.884^{\mathrm{C}}$ \\
\hline FAZ area (mean $\pm \mathrm{SD}), \mathrm{mm}^{2}$ & $0.37 \pm 0.11$ & $0.4 \pm 0.16$ & $0.03 \pm 0.1$ & $8.1 \%$ & $0.041^{\mathrm{C}}$ \\
\hline FD Full (mean \pm SD) & $1.57 \pm 0.04$ & $1.54 \pm 0.04$ & $-0.02 \pm 0.05$ & $-1.3 \%$ & $0.003^{\mathrm{C}}$ \\
\hline FD SCP (mean \pm SD) & $1.54 \pm 0.04$ & $1.52 \pm 0.04$ & $-0.02 \pm 0.05$ & $-1.3 \%$ & $0.021^{\mathrm{c}}$ \\
\hline FD DCP $($ mean \pm SD) & $1.57 \pm 0.04$ & $1.54 \pm 0.05$ & $-0.03 \pm 0.05$ & $-1.9 \%$ & $<0.001^{\mathrm{C}}$ \\
\hline VD Full (mean \pm SD), \% & $31.3 \pm 5.58$ & $28.8 \pm 5.18$ & $-2.5 \pm 5.72$ & $-8 \%$ & $0.01^{\mathrm{c}}$ \\
\hline VD SCP (mean \pm SD), \% & $27.8 \pm 4.09$ & $25.3 \pm 4.78$ & $-2.53 \pm 5.13$ & $-9.1 \%$ & $0.003^{\mathrm{C}}$ \\
\hline VD DCP (mean \pm SD), \% & $28.5 \pm 5.28$ & $25.5 \pm 5.96$ & $-3.03 \pm 6.24$ & $-10.6 \%$ & $0.004^{\mathrm{C}}$ \\
\hline Skeleton VD Full (mean \pm SD), $\%$ & $9 \pm 1.99$ & $7.8 \pm 1.76$ & $-1.2 \pm 2.32$ & $-13.3 \%$ & $0.003^{\mathrm{C}}$ \\
\hline Skeleton VD SCP (mean \pm SD), \% & $7.77 \pm 1.6$ & $6.8 \pm 1.62$ & $-0.97 \pm 2.16$ & $-12.5 \%$ & $0.007^{\mathrm{C}}$ \\
\hline Skeleton VD DCP (mean \pm SD), \% & $8.9 \pm 1.8$ & $7.44 \pm 2.03$ & $-1.45 \pm 2.36$ & $-16.3 \%$ & $<0.001^{\mathrm{C}}$ \\
\hline
\end{tabular}

Abbreviations: CDVA, corrected distance visual acuity; CMT, central macular thickness; DCP, deep capillary plexus; FAZ, foveal avascular zone; FD, fractal dimension; Full, full retinal thickness; IOP, intraocular pressure; LogMAR, Logarithm of the minimum angle of resolution; PFT, parafoveal thickness; SCP, superficial capillary plexus; VD, vascular density.

a Statistically significant if $<0.05$

b Wilcoxon signed rank test

${ }^{\mathrm{c}}$ Paired t test

Table 3: Quantitative analysis and comparison of the pre and post-treatment results of the 3X3 mm scan group 


\begin{tabular}{|l|l|l|l|l|l|}
\hline Metric & Pre-Treatment & Post-Treatment & Change & $\%$ Change & $p^{\text {-value }}$ \\
\hline LogMAR CDVA (mean \pm SD) & $0.63 \pm 0.34$ & $0.51 \pm 0.38$ & $-0.12 \pm 0.24$ & $-19 \%$ & $0.122^{\mathrm{b}}$ \\
\hline CMT (mean \pm SD), $\mu \mathrm{m}$ & $370 \pm 69$ & $276 \pm 51$ & $-93 \pm 74$ & $-25.1 \%$ & $0.008^{\mathrm{b}}$ \\
\hline PFT (mean \pm SD), $\mu \mathrm{m}$ & $384 \pm 34$ & $331 \pm 25$ & $-53 \pm 42$ & $-13.8 \%$ & $0.005^{\mathrm{c}}$ \\
\hline IOP (mean \pm SD), mmHg & $17.8 \pm 1.9$ & $15.7 \pm 2.7$ & $-2.5 \pm 2.6$ & $-14 \%$ & $0.064^{\mathrm{c}}$ \\
\hline Signal Strength (mean \pm SD) & $5.9 \pm 1.4$ & $6.3 \pm 1.1$ & $0.4 \pm 0.9$ & $6.8 \%$ & $0.169^{\mathrm{c}}$ \\
\hline FAZ area (mean \pm SD), mm ${ }^{2}$ & $0.36 \pm 0.09$ & $0.39 \pm 0.12$ & $0.03 \pm 0.04$ & $8.3 \%$ & $0.067^{\mathrm{c}}$ \\
\hline FD Full (mean \pm SD) & $1.53 \pm 0.03$ & $1.49 \pm 0.04$ & $-0.04 \pm 0.05$ & $-2.6 \%$ & $0.046^{\mathrm{c}}$ \\
\hline FD SCP (mean \pm SD) & $1.48 \pm 0.05$ & $1.43 \pm 0.06$ & $-0.05 \pm 0.05$ & $-3.4 \%$ & $0.01^{\mathrm{c}}$ \\
\hline FD DCP (mean \pm SD) & $1.53 \pm 0.04$ & $1.49 \pm 0.05$ & $-0.04 \pm 0.06$ & $-2.6 \%$ & $0.073^{\mathrm{c}}$ \\
\hline VD Full (mean \pm SD), \% & $29.5 \pm 5.97$ & $26.1 \pm 7.67$ & $-3.38 \pm 4.08$ & $-11.5 \%$ & $0.038^{\mathrm{c}}$ \\
\hline VD SCP (mean \pm SD), \% & $22.9 \pm 6.17$ & $19.7 \pm 6.3$ & $-3.27 \pm 3.18$ & $-14.3 \%$ & $0.015^{\mathrm{c}}$ \\
\hline VD DCP (mean \pm SD), \% & $26.2 \pm 6.22$ & $23.9 \pm 7.32$ & $-2.3 \pm 3.95$ & $-8.8 \%$ & $0.118^{\mathrm{c}}$ \\
\hline Skeleton VD Full (mean \pm SD), \% & $6.93 \pm 1.4$ & $5.65 \pm 1.6$ & $-1.28 \pm 1.75$ & $-18.5 \%$ & $0.059^{\mathrm{c}}$ \\
\hline Skeleton VD SCP (mean \pm SD), \% & $5.45 \pm 1.6$ & $4.09 \pm 1.3$ & $-1.37 \pm 1.2$ & $-25.1 \%$ & $0.009^{\mathrm{c}}$ \\
\hline Skeleton VD DCP (mean \pm SD), \% & $6.86 \pm 1.6$ & $5.52 \pm 1.5$ & $-1.35 \pm 1.9$ & $-19.7 \%$ & $0.066^{\mathrm{c}}$ \\
\hline
\end{tabular}

Abbreviations: CDVA, corrected distance visual acuity; CMT, central macular thickness; DCP, deep capillary plexus; FAZ, foveal avascular zone; FD, fractal dimension; Full, full retinal thickness; IOP, intraocular pressure; LogMAR, Logarithm of the minimum angle of resolution; PFT, parafoveal thickness; SCP, superficial capillary plexus; VD, vascular density.

a Statistically significant if $<0.05$

${ }^{\mathrm{b}}$ Wilcoxon signed rank test

${ }^{c}$ Paired t test

\section{Figures}




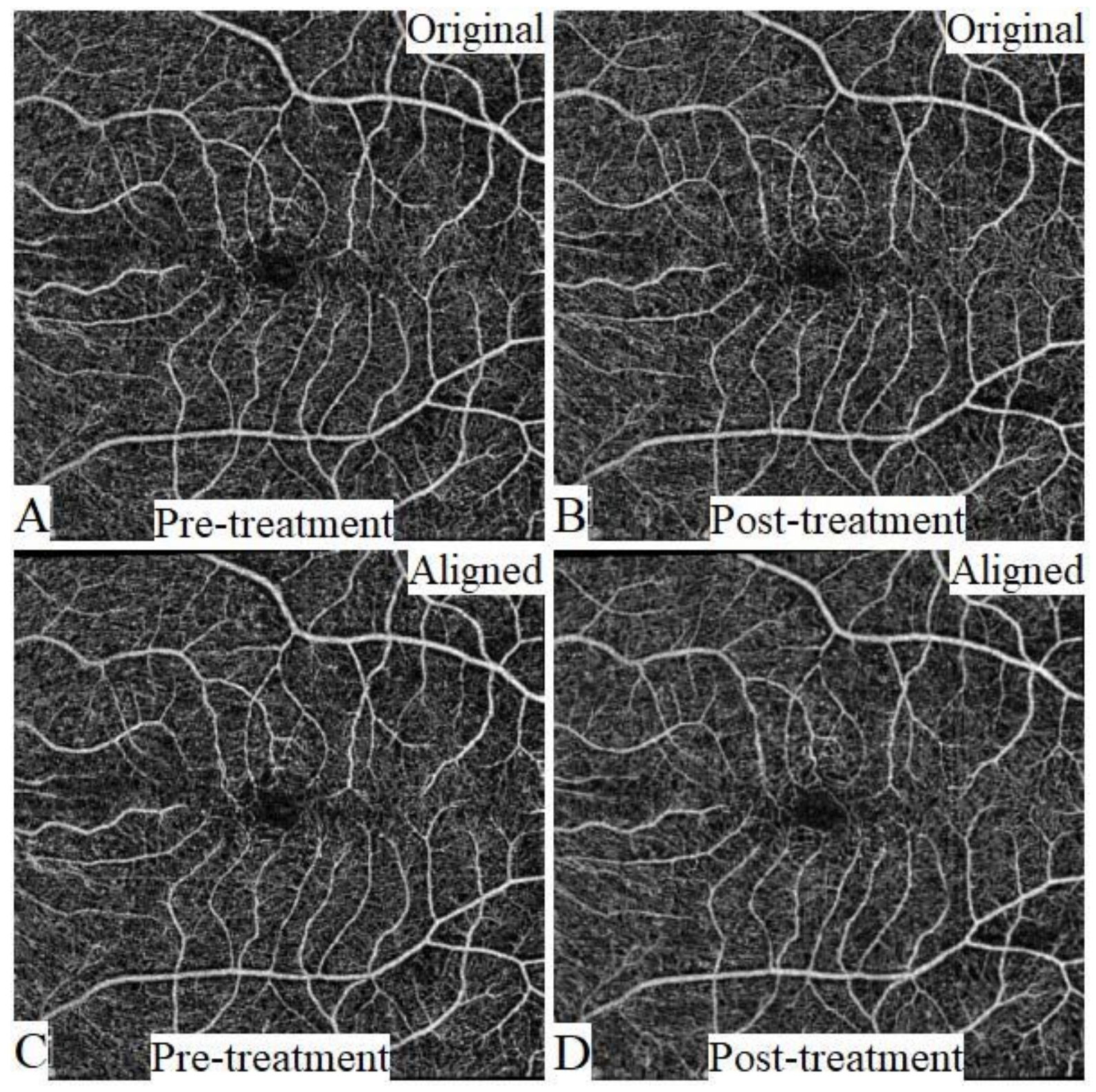

Figure 1

Automated image alignment. Original $6 \times 6 \mathrm{~mm}$ pre and post-treatment images ( $A$ and $B$ ) were automatically registered and aligned ( $C$ and $D)$ using a commercially available retina alignment software to allow comparison of only areas common to both images. 

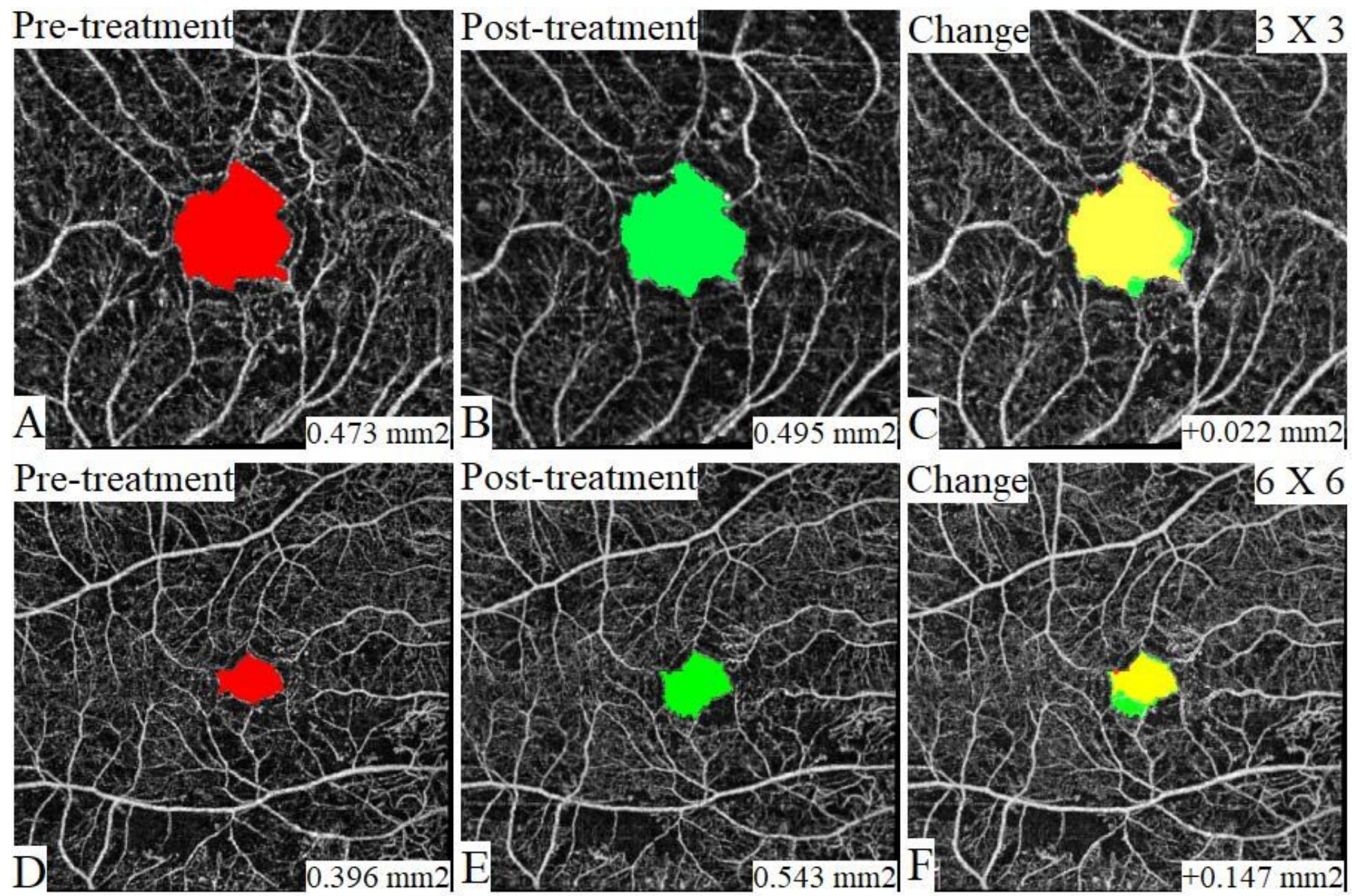

Figure 2

FAZ area measurement. The FAZ area was measured manually using Image $J$ for the pre and posttreatment $3 \times 3 \mathrm{~mm}(A$ and $B$ ) and $6 \times 6 \mathrm{~mm}$ ( $D$ and $E$ ) macular scan images. FAZ area change between pre and post-treatment images was then calculated ( $\mathrm{C}$ and $\mathrm{F})$. 

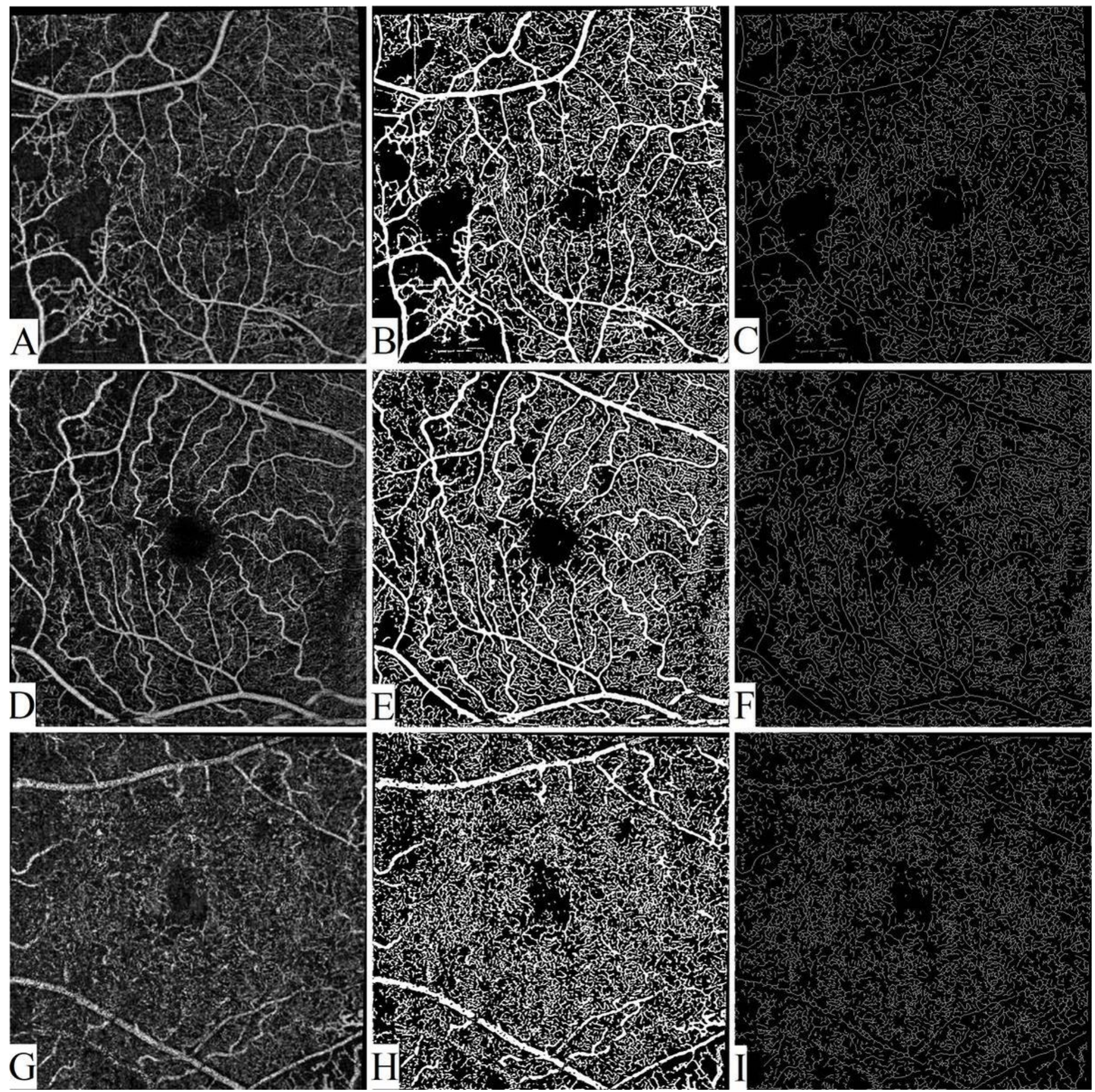

Figure 3

Image processing technique. En-face OCTA images $(A, D$, and $G)$ were converted into a binary image (B, $E$, and $\mathrm{H}$ ) by using a combined method consisting of a global threshold, hessian filter, and adaptive threshold in MATLAB. Skeletonized images were created by deleting the pixels in the outer boundary of the binarized, white-pixelated vessels until only 1 pixel remained along the width of the vessels $(C, F$, and I). 

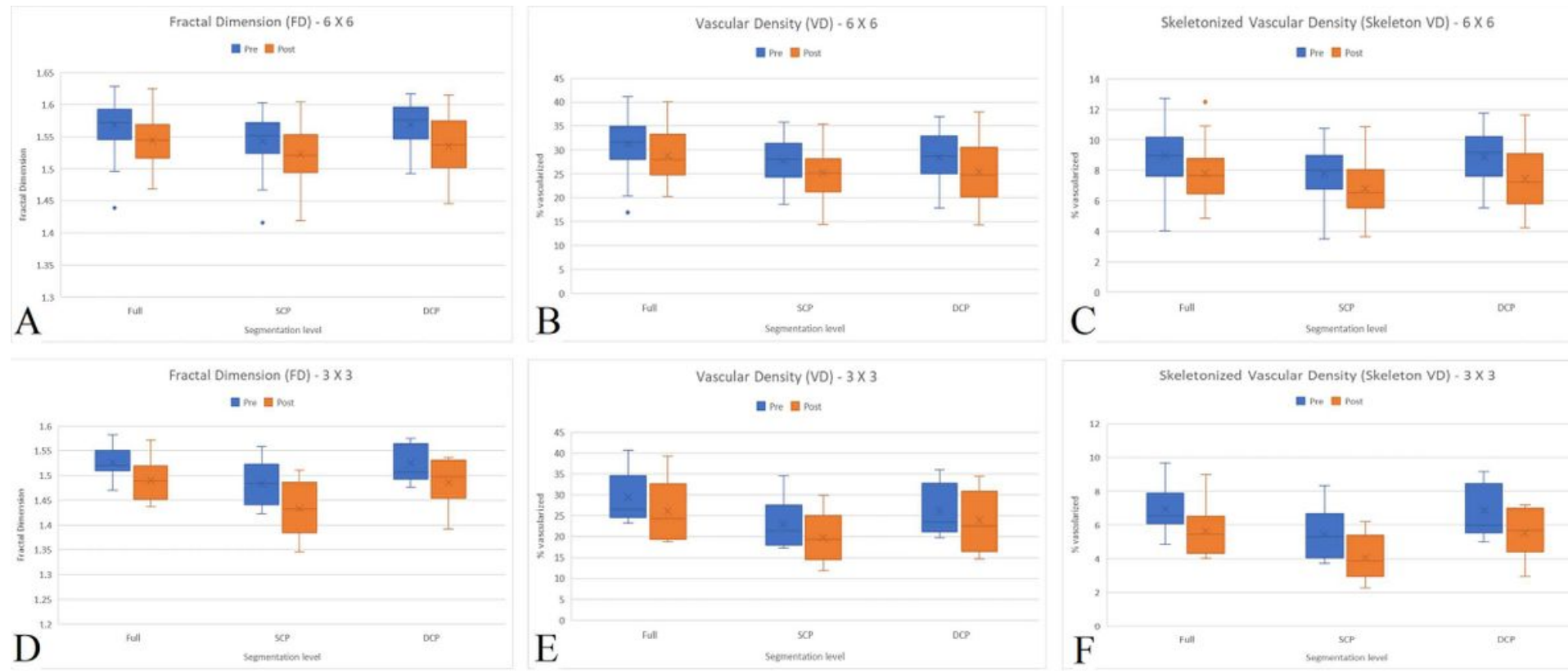

B

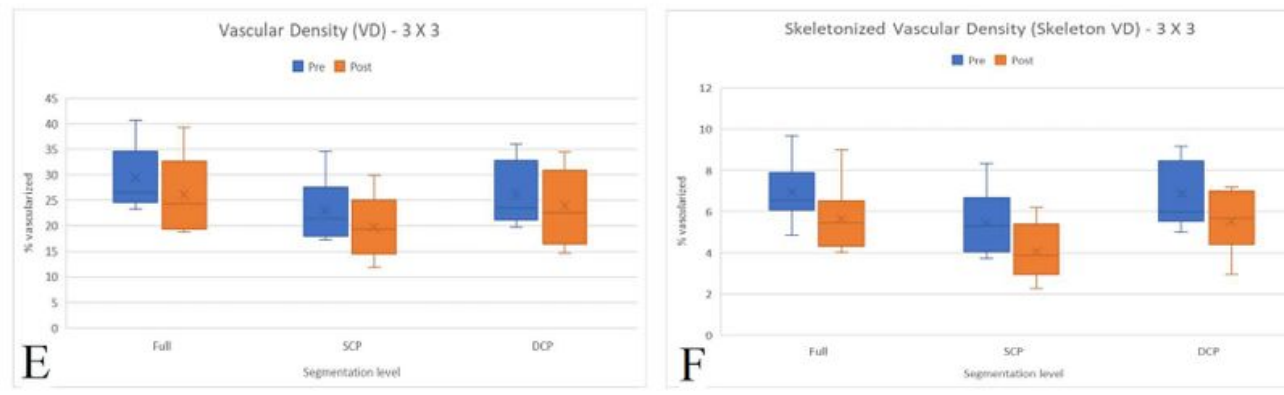

\section{Figure 4}

Boxplots of pre and post-treatment values from both the $6 \times 6$ and $3 \times 3 \mathrm{~mm}$ scan protocols at different segmentation levels. In the $6 \times 6 \mathrm{~mm}$ scan group, there was a statistically significant decrease in the FD, VD, and skeleton VD of Full, SCP, and DCP (A, B, and C). In the $3 X 3 \mathrm{~mm}$ scan group, there was a statistically significant decrease in the FD and VD Full and SCP, and the skeleton VD-SCP. There was a decrease in skeleton VD-Full, and FD, VD, and skeleton VD-DCP but these were not statistically significant (D, E, and F).
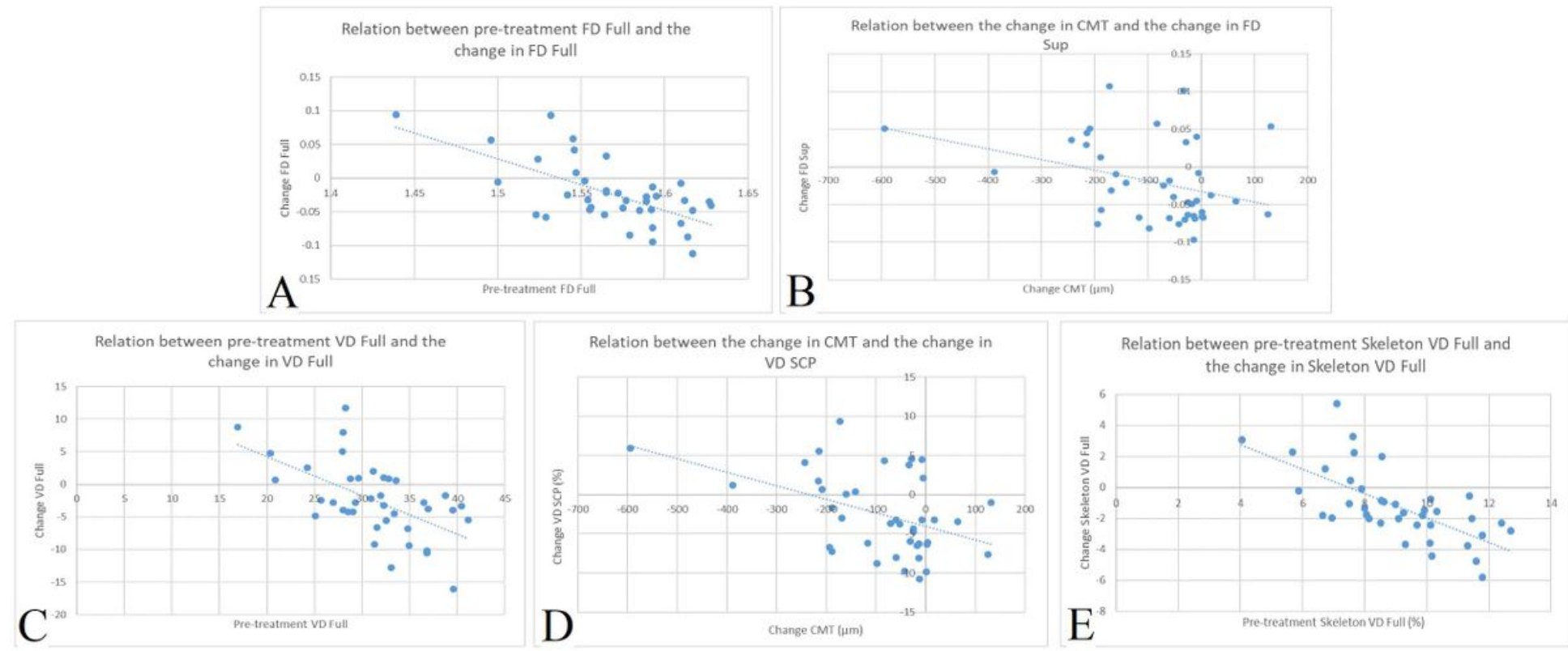

Figure 5

Examples of scatter plots for factors significantly associated with post-treatment changes using univariate analysis. There was a significant negative correlation between (A) pre-treatment FD Full and 
the change in FD Full $(r=-0.541, p<0.001)$, (B) the change in CMT and the change in FD SCP $(r=-0.324$, $p=0.042)$, (C) pre-treatment VD Full and the change in VD Full $(r=-0.560, p<0.001)$, (D) the change in CMT and the change in VD SCP $(r=-0.389, p=0.013)$, (E) and pre-treatment skeleton VD Full and the change in skeleton VD Full $(r=-0.694, p<0.001)$.

\section{Supplementary Files}

This is a list of supplementary files associated with this preprint. Click to download.

- CONSORT2010ChecklistAymanElnahry.doc 